\title{
Assessment of Power Losses of an Inverter-Driven Induction Machine With Its Experimental Validation
}

\author{
Carlos A. Hernandez-Aramburo, Member, IEEE, Tim C. Green, Senior Member, IEEE, and \\ Sandy Smith, Senior Member, IEEE
}

\begin{abstract}
Detailed power loss distribution in induction machines is assessed using time-stepped finite-element analysis coupled to circuit equations of an inverter. Losses are examined for various load conditions. Iron losses are largely unexplored and so particular attention is paid to them here. The simulation has revealed the division between ohmic, hysteresis, classic eddy-current, and anomalous losses; and the distribution in the frequency spectrum between fundamental, slotting, and multiples of the switching frequency. Insight is also gained into the spatial location of the loss. Experimental validation is provided for several fundamental frequencies from full-load to light-load conditions.
\end{abstract}

Index Terms-Efficiency, finite element (FE), inverter-driven induction machines, iron losses, power losses, variable-speed drives.

\section{INTRODUCTION}

A S NEW manufacturing standards take effect, induction motor designers face more challenging machine efficiency specifications [1]. Power losses must then be thoroughly understood and predicted. In order to accurately calculate the power losses in a machine, two largely overlooked aspects are acquiring special importance: iron losses and stray losses.

Iron loss calculation is still the most complex problem when estimating the power losses in an induction machine. There are a number of reasons for the difficulty in achieving good results, among them are the following.

- The loss mechanism is still not well understood even in laminations of simple shapes.

- Second-order effects (such as the eddy currents) are very difficult to isolate and measure.

- The physical properties of iron laminations can vary dramatically from one sample to another.

Paper IPCSD 03-020, presented at the 2002 Industry Applications Society Annual Meeting, Pittsburgh, PA, October 13-18, and approved for publication in the IEEE TRANSACTIONS ON INDUSTRY APPLICATIONS by the Electric Machines Committee of the IEEE Industry Applications Society. Manuscript submitted for review September 19, 2002 and released for publication March 22, 2003. This work was supported by the Consejo Nacional de Ciencia y Tecnología-México under Grant 115297.

C. A. Hernandez-Aramburo and T. C. Green are with Electrical Engineering, Imperial College, London SW7 2BT, U.K. (e-mail: c.hernandez@ic.ac.uk; t.green@ic.ac.uk).

S. Smith is with Electrical Engineering, University of Manchester Institute of Science and Technology, Manchester M60 1QD, U.K. (e-mail: sandy.smith@umist.ac.uk).

Digital Object Identifier 10.1109/TIA.2003.813744
- During motor manufacturing, the iron laminations are exposed to a number of mechanical stresses (such as punching, heating and assembly) which change their magnetic properties.

Not withstanding of these difficulties, considerable efforts are being made by industry and academia to understand iron loss phenomena in quantitatively and qualitatively terms.

Statistical approaches have been used [2] in which experimental iron loss data are correlated to the physical construction details of the machine. In general, statistical approaches have important advantages: the contribution of each design parameter to the total iron power loss is weighed and the computational effort to obtain iron loss estimates is small. However, serious disadvantages have to be mentioned: they require a large database of motors containing intrinsic construction details of each machine and a lot of experimentation must be done in order to find a reliable correlation between losses and the machine design parameters.

Another approach to describe the iron losses in an induction machine is to rely on the well-established equivalent circuit model. One of the earliest references discussing iron losses in this model might be attributed to Behrend, in 1896 [3]. When secondary effects are to be taken into account, the model can be extended with a chain of LR-branches to consider higher order harmonics, extra resistors to account for eddy-current power dissipation, and/or saturable inductors (where more recent references [4], [5], and [6] are but a representative sample). Models based on the equivalent circuit achieve some success by quantitatively describing iron losses; however, they fail to provide information on where they are concentrated in the machine.

The physical distribution of losses may be approximated by creating an analytical model of a simplified geometry of the machine [7]. However, some important construction features may be overlooked, such as the dimension of tooth tips, for instance.

Under the penalty of the being computationally demanding, finite-element $(F E)$ techniques may be the best approach to estimate and provide insight into iron losses.

This paper addresses the power loss distribution in induction machines using a time-step FE technique coupled to equivalent circuit equations in which an inverter model is embedded. Engineering knowledge is advanced by explaining in detail the power loss behavior of an inverter-fed induction machine under different operating conditions. 


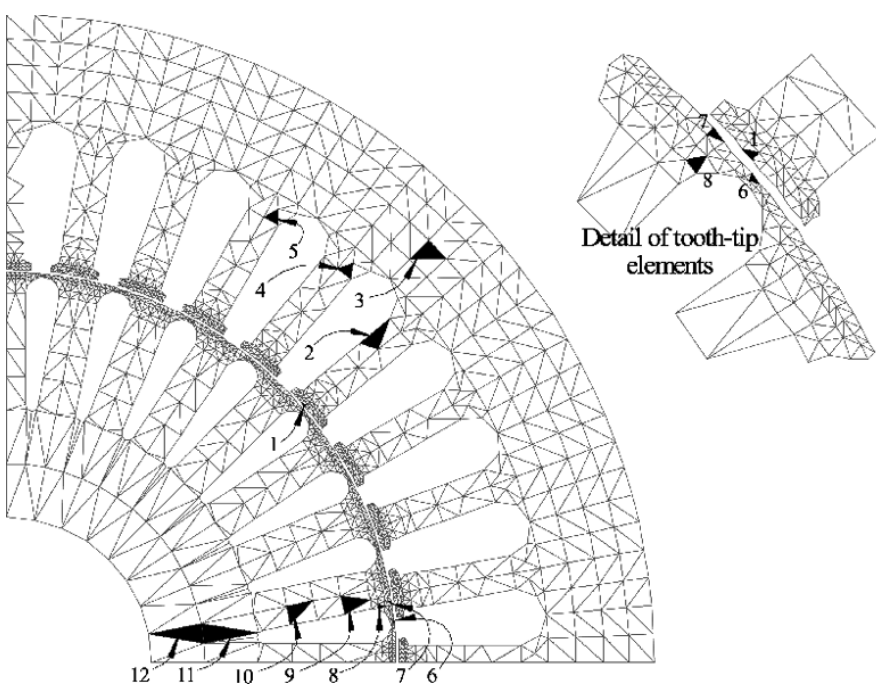

Fig. 1. Iron meshes with certain reference finite elements.

\section{SYSTEM REPRESENTATION}

The motor-inverter system is represented by integrated simulation governed by (1) and (2)

$$
\begin{aligned}
\frac{\mathrm{d}}{\mathrm{d} \mathbf{t}}[\mathbf{\Psi}] & =[\mathbf{V}]-[\mathbf{F}(\mathbf{I})]-[\mathbf{R}][\mathbf{I}] \\
{[\mathbf{I}] } & =[\mathbf{L}]^{-1}[\mathbf{\Psi}] .
\end{aligned}
$$

The machine model is similar to that described in previous research [8], [9]: the stator variables are in the $A B C$ reference frame, while a $d q$ model is employed to represent the rotor; $\mathbf{R}$ is the resistance matrix; $\mathbf{I}$, the phase currents vector; $\Psi$, the flux linkages, and $\mathbf{L}$ is the inductance matrix. The motor nonlinearities are accounted for by continuously recomputing $\mathbf{L}$ by $\mathrm{FE}$ analysis. A natural $A B C$ reference frame was used in the stator to accommodate the voltage drops in the inverter and to preserve the measurable stator currents and voltages as a direct solution of it. Although this appears to require an additional equation, in reality, simple electrical phase symmetry may be invoked to reduce the phase variables from three to two. The rotor on the other hand has no natural phase reference, so the standard $d q$ variables are used to keep the simulation time to a minimum. Fig. 1 shows the FE mesh of the stator and rotor laminations, and the inset shows a close-up of the elements near the air gap. Two important modifications have been made to this model [10]: the phase voltages $(\mathbf{V})$ are inverter switching waveforms, and the nonlinear voltage drops across the inverter transistors and diodes are represented by $\mathbf{F}(\mathbf{I})$. This inverter-motor model was used to simulate the machine at several combinations of fundamental frequency and mechanical load.

\section{POWER LOSS DISTRIBUTION}

The inverter and machine were simulated for all combinations of four fundamental frequencies $(5,10,25$, and $50 \mathrm{~Hz})$ and four load conditions (represented as four different unnormalized slips of $0.5,1.25,2.0$, and $2.5 \mathrm{~Hz}$ ). Hereafter, the slips of 0.5 and $2.5 \mathrm{~Hz}$ will be referred to as the light-load and full-load conditions, respectively. In all cases the switching frequency was set to $5 \mathrm{kHz}$. The inverter dc-link voltage was set to $400 \mathrm{~V}$ to provide an output voltage of $240 \mathrm{~V} \mathrm{rms}$ at $50 \mathrm{~Hz}$, which is the rated voltage for the motor under test.

The corresponding stator voltage for each fundamental frequency follows an open-loop V/F control strategy. This strategy was chosen because of its widely spread use in industry, its well know operation principle, and it may be regarded as a benchmark for more complex closed-loop control schemes. Since constant flux is a necessary condition for fair comparison of the iron losses for different fundamental frequencies, a voltage-boosting strategy was implemented to compensate for the effects of the voltage drop across the stator resistance against the fundamental frequency, which is particularly important at low frequencies. In addition to these inverter-fed simulations, a set of sinusoidal-excited simulations for the same slips are included as a benchmark.

The power loss calculation was applied in a post-processing stage to the FE simulation data and the results obtained for the stator and rotor are discussed in the following sections.

\section{A. Iron Loss Model}

Although there are several variants of the calculation of the three contributions to iron loss power density, one widely accepted set of expressions is given by (3)-(6) [11], [12]

$$
\begin{aligned}
P_{\text {iron }} & =P_{\text {hys }}+P_{\text {eddy }}+P_{\text {anom }} \\
P_{\text {hys }} & =k_{1 h y s} f B_{p k}^{k_{2 h y s}}\left(1+\frac{k_{3 h y s}}{B_{p k}} \sum_{i}^{N} \Delta B_{i}\right) \\
P_{\text {eddy }} & =\frac{\sigma \delta^{2}}{12} \frac{1}{T} \int_{T}\left(\frac{d B}{d t}\right)^{2} d t \\
P_{\text {anom }} & =\frac{k_{\text {anom }}}{T} \int_{T}\left|\frac{d B}{d t}\right|^{3 / 2} d t
\end{aligned}
$$

where

$P_{\text {iron }} \quad$ iron power loss density $\left(\mathrm{W} / \mathrm{m}^{3}\right)$;

$P_{\text {hys }} \quad$ hysteresis loss density $\left(\mathrm{W} / \mathrm{m}^{3}\right)$;

$P_{\text {eddy }}$ classic eddy-current loss density $\left(\mathrm{W} / \mathrm{m}^{3}\right)$;

$P$ anom anomalous loss density $\left(\mathrm{W} / \mathrm{m}^{3}\right)$;

$B, B_{p k}$ flux density, peak flux density, respectively (T);

$\Delta B_{i} \quad$ amplitude of the $i$ th minor loop (T);

$N \quad$ number of minor loops;

$T$ period of the waveform (s);

$k_{1 \text { hys }} \quad$ hysteresis loss linear constant;

$k_{2 h y s} \quad$ hysteresis loss exponential constant;

$k_{3 \text { hys }} \quad$ hysteresis loss minor loops constant;

$k_{\text {anom }}$ anomalous loss constant;

$\sigma \quad$ lamination conductivity $(\mathrm{S} / \mathrm{m})$;

$\delta \quad$ lamination thickness $(\mathrm{m})$.

The values of the constants in (4)-(6) cannot be included here because they are commercially confidential.

An important feature of (4) is the term enclosed in parenthesis, which corresponds to the Lavers' Correction Factor $(L C F)$ as presented in [13]. This term is an approximation to account for hysteresis losses produced by the minor loops that a BH-locus may contain.

Undoubtedly, there are other models for iron losses which may result in more accurate predictions [14], [15], [16]. The 


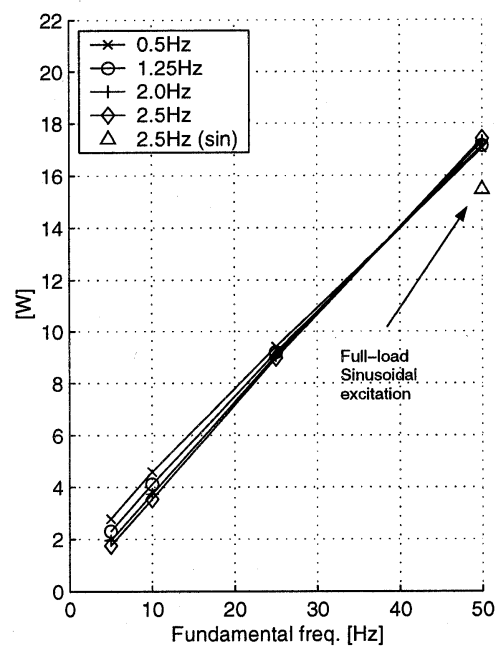

Fig. 2. Stator hysteresis power loss.

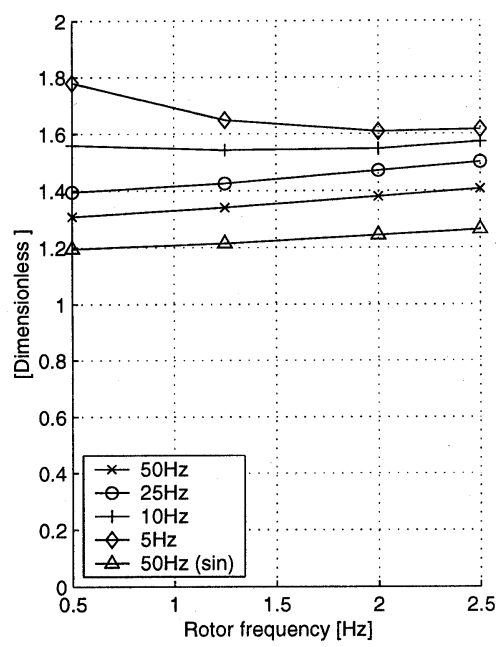

Fig. 3. LCF for the stator.

model represented by the above was chosen because of its reasonable accuracy and invaluable experience gained in the past [9], [17].

\section{B. Iron Power Loss in the Stator}

The simulation results obtained for hysteresis power losses are, perhaps, the simplest case to describe as they seem to behave linearly against the fundamental frequency (Fig. 2), which is in accordance with the chosen model (4). Fig. 2 also indicates a marginal change of power loss with the motor load. Fig. 3 shows the impact of the LCF on the total hysteresis loss as a function of the slip. For pulsewidth-modulation (PWM) excitation, the minor-loop correction represents between 30\%-80\% of the hysteresis loss calculated without it. For the sinusoidal case, the correction is only about $20 \%$.

Figs. 4 and 5 show the classic eddy-current loss and the anomalous loss in the stator, respectively.

The behavior of the classic eddy-current loss in the stator is surprisingly linear against frequency. Equation (5) indicates that

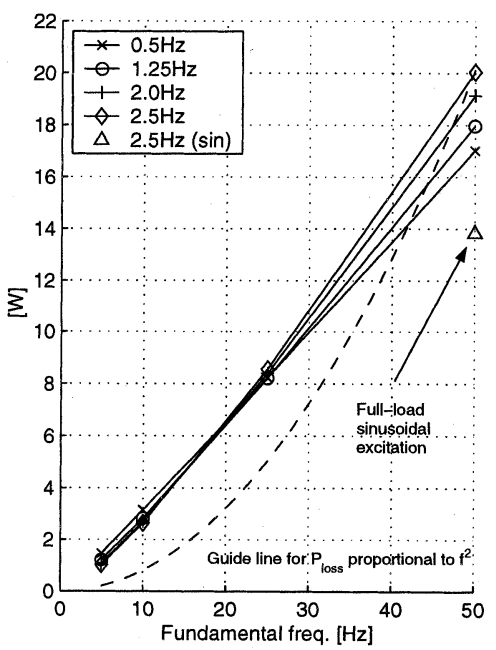

Fig. 4. Stator classic eddy-current loss.

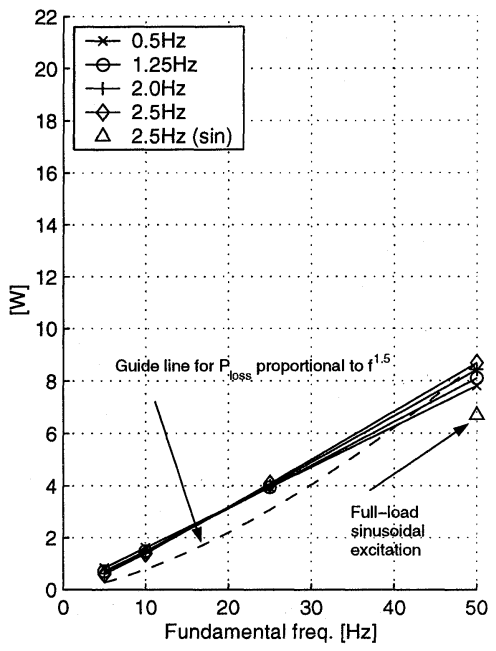

Fig. 5. Stator anomalous loss.

the eddy-current power loss should be a quadratic function of frequency, such as that shown as a dashed line in the same figure for a slip of $2.5 \mathrm{~Hz}$. A frequency spectrum analysis revealed that the difference was a consequence of a chain of events described as follows.

- As the fundamental frequency decreases, a low modulation index must be used to keep the mutual flux constant.

- A low modulation index significantly increases the magnitude of the sideband components around the second multiple of the switching frequency with respect to the fundamental frequency component.

- Because of their relative high frequency (approximately twice the switching frequency), these second-order sidebands have a considerable impact on iron loss.

- The impact of the second-order sidebands, added to the contribution of the sidebands around the switching frequency, is important enough to break the "expected" quadratic behavior of the eddy-current losses against the fundamental frequency. 


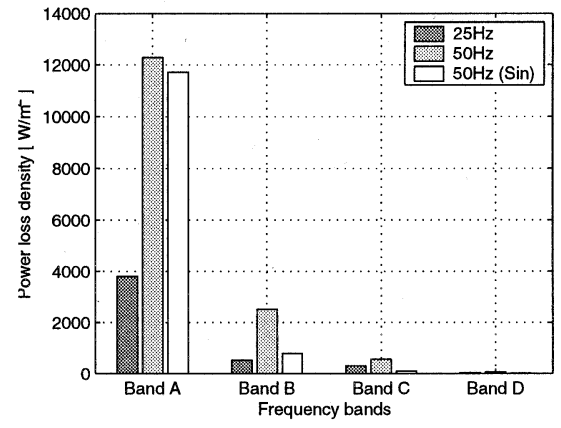

(a)

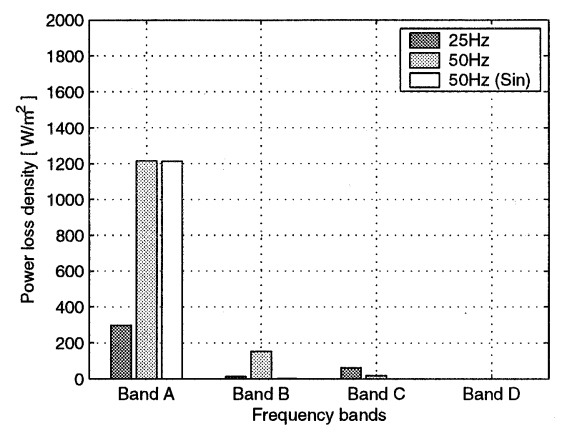

(c)

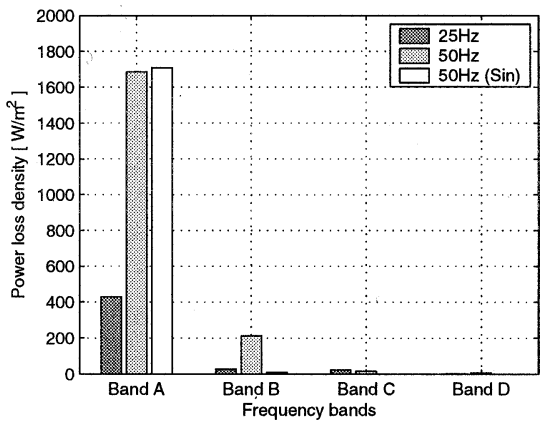

(b)

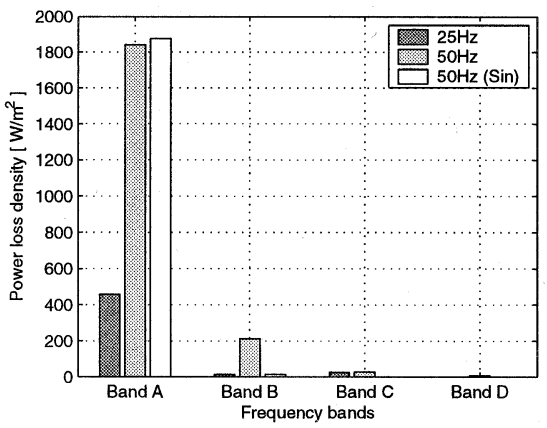

(d)

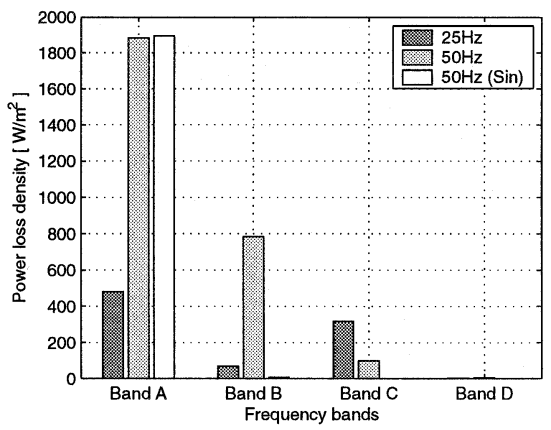

(e)

Fig. 6. Frequency partition of the power loss density in various elements of the stator mesh. (a) Element 1: tooth tip, (b) Element 2: middle of the tooth. (c) Element 3: back iron. (d) Element 4: tooth base. (e) Element 5: tooth base in phase-band border.

To provide a clearer perspective on the contribution of the PWM frequency components to iron loss, a power loss partition in frequency is performed. The frequency spectrum is divided into four regions: band $A(0-2.5 \mathrm{kHz})$ contains the fundamental frequency and the most significant slotting harmonics; band $B$ $(2.5-7.5 \mathrm{kHz})$, the sidebands of the switching frequency; band $C$ $(7.5-12.5 \mathrm{kHz})$, the sideband components of the second multiple of the switching frequency; and finally, band $D(12.5-17.5 \mathrm{kHz})$ includes the sideband components of the third multiple of the switching frequency.

Fig. 6 shows the classic eddy power loss density distribution for the chosen frequency bands for the five stator elements marked in Fig. 1. The power loss density is shown for two fundamental frequencies $(25$ and $50 \mathrm{~Hz})$ under PWM excitation at a rotor slip of $2.5 \mathrm{~Hz}$. A sinusoidal excitation case at the same slip is also shown as benchmark. The first aspect to note is that the location of an FE within the iron lamination is remarkably important. Element 1 (in the tooth tip) has the highest loss density. This is because its flux density is higher than any other element and its $\partial \mathbf{B} / \partial t$ (in both radial and tangential directions) is the highest. The power densities for the rest of the elements are about the same order (note that the $y$ axis has the same scale in Fig. 6(b)-(e) but Fig. 6(a) is different).

Band D is only included in this figure to show how little contribution to iron loss was predicted from the sidebands around the third multiple of the switching frequency.

By comparing the power loss density in band A for all elements, it may be observed that it changes approximately as a quadratic function between the $25-$ and $50-\mathrm{Hz}$ cases (a factor of 4 for double the frequency). The difference in power loss density between the sinusoidal case and the $50-\mathrm{Hz}$ PWM case is only marginal. This was expected because band A does not contain any significant PWM sideband components.

There is no obvious pattern of change, however, for the loss density contained in band B between the $25-$ and $50-\mathrm{Hz}$ cases. For element 1, the $50-\mathrm{Hz}$ sinusoidal case is not zero; this reveals that there are some slotting harmonics in this band. If this power loss density is subtracted from the $50-\mathrm{Hz}$ PWM case, the ratio between the remaining loss at $50-\mathrm{Hz} \mathrm{PWM}$ and the $25-\mathrm{Hz}$ case 
is about 3.5. For elements $2-5$, this ratio is different at approximately $8,12,14$, and 12 , respectively.

The loss density in band $\mathrm{C}$ shows a large decrease for an increase in fundamental frequency in elements 3 and 5 . This can be linked with the decrease in magnitude of the sidebands around the second multiple of the switching frequency as the modulation index increases. However, this explanation does not apply for the loss density in this band in elements 1,2, and 4.

Although there is no obvious pattern for how the power loss density for PWM-related harmonics change with fundamental frequency, it is important to note that, in general, it does not follow the same rules as the fundamental contribution itself (band A). Clearly, a quadratic behavior of the classic eddy-current loss against the fundamental frequency for the whole stator (as the one shown with a dashed line in Fig. 4) should not be expected. The quadratic function only accounts for the fundamental frequency and ignores the effects of the PWM-related harmonics and their nonuniform spatial distribution in the stator laminations.

Fig. 6 also allows an important comparison between elements 4 and 5. These two elements might be expected to dissipate the same power as they have symmetrical locations in the iron laminations, being displaced by one slot. The loss densities in band A are only marginally different while in bands B and C the loss density in element 5 is considerably larger than in element 4. The higher susceptibility to loss of element 5 is because it has a larger magnetic flux. Element 5 is in a tooth located between two phase bands and therefore it has all the additional phase-band leakage flux produced by the PWM power source, which increases the power dissipation in this element.

For the preceding power loss analysis in the frequency domain, all the frequency components below a certain threshold were neglected. The threshold for each element was defined in terms of its own fundamental component of flux density. Any frequency component smaller than $0.03 \%$ of the fundamental component was neglected. The components below this threshold occur across a wide frequency range and are believed to originate from small numerical errors in the time-step solutions. As such they make a false contribution to the iron losses and should be excluded.

The behavior of anomalous loss [calculated from (6)] against the fundamental frequency is shown in Fig. 5. The general trend for the anomalous loss has many similarities with the classic eddy-current loss as they both share the term $\partial \mathbf{B} / \partial t$ raised to some power (power 2 for classical loss and $3 / 2$ for anomalous loss). Again, the behavior against the fundamental frequency is surprisingly linear due to the contribution from the first- and second-order PWM sideband components at low modulation indexes, as already explained for the case of the eddy-current loss. The impact of these high-order PWM sidebands in the anomalous loss may be evaluated by comparing the results for the power loss dissipation at a rotor slip of $2.5 \mathrm{~Hz}$ with the dashed trace in Fig. 5, which is a guide line changing as $f^{3 / 2}$.

\section{Iron Power Loss in the Rotor}

The factors which are significant for the hysteresis power loss in the rotor are different to those in the stator. As Fig. 7

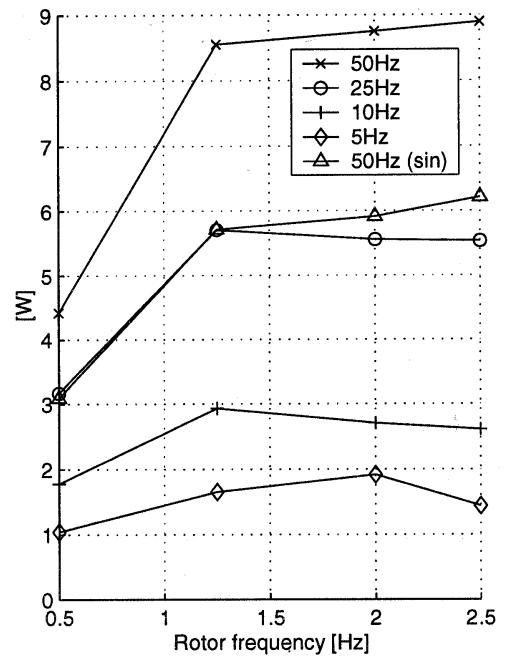

Fig. 7. Rotor hysteresis power loss.

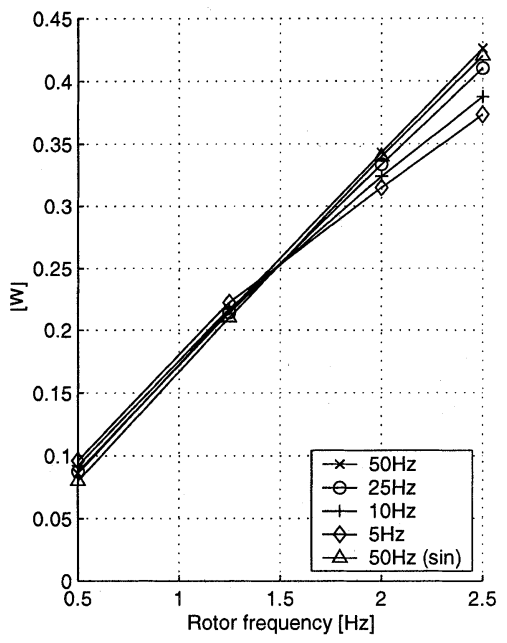

Fig. 8. Rotor hysteresis power loss without LCF.

shows, the losses remain basically constant against the rotor frequency from about 1.25 to $2.5 \mathrm{~Hz}$ and sharply decrease as the rotor comes closer to the synchronous speed. Without the LCF, hysteresis losses show a very different structure, as shown in Fig. 8. The first difference to point out is that the magnitude of the losses predicted using the LCF is much larger than those without it. For a fundamental frequency of $50 \mathrm{~Hz}$ and a rotor frequency of $0.5 \mathrm{~Hz}$, the LCF works out to be $4.5 / 0.9=50$; at the other extreme, for a fundamental frequency of $5 \mathrm{~Hz}$ and a rotor frequency of $2.5 \mathrm{~Hz}$ the correction is about $1.5 / 0.37 \approx 4$. These numbers indicate that the LCF contribution for the hysteresis loss calculation is considerably higher and spread over a larger range for the rotor than for the stator. The second major difference between Figs. 7 and 8 is that the latter shows that hysteresis loss is dependent on the rotor frequency and almost independent of the stator fundamental frequency. This can be explained by considering that expression (4) without the LCF estimates hysteresis losses as a linear function of the frequency of the flux density present in the lamination (the rotor frequency) and it is independent of the stator fundamental frequency. By 


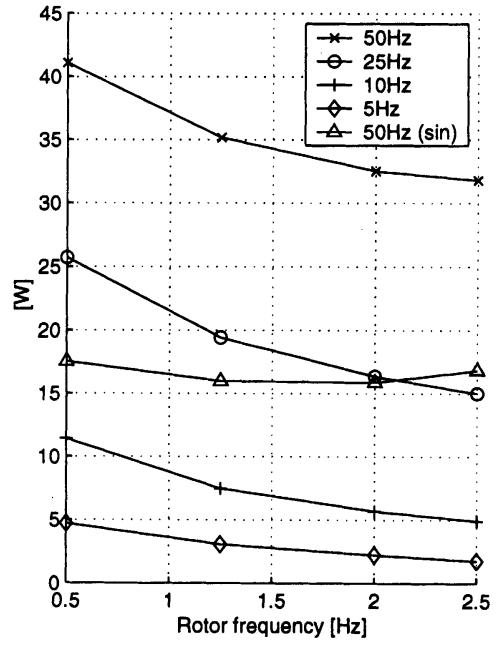

Fig. 9. Rotor eddy-current loss.

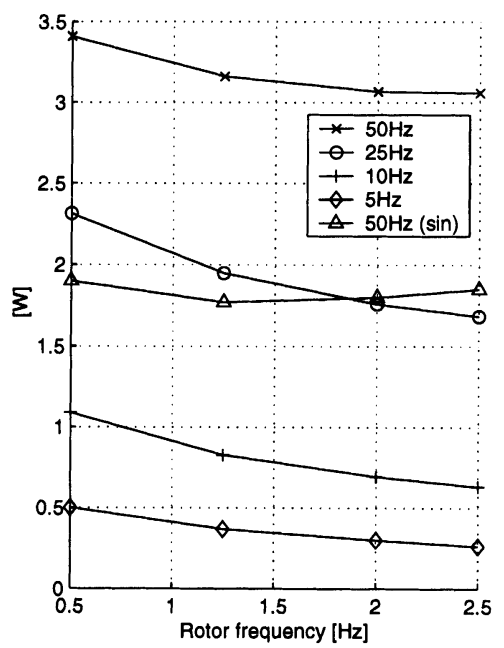

Fig. 10. Rotor anomalous loss.

bringing the LCF into the calculation, additional causes of loss are considered, such as slotting and PWM-related frequencies. In particular, slotting accounts for the strong dependency on the fundamental frequency shown in Fig. 7.

Fig. 9 shows the effect of the motor load on classic eddycurrent loss: as the load increases, the rotor classic eddy loss decreases. This can be linked with the rotor saturation. As the load increases, some parts of the rotor laminations (the leakage paths) become more saturated and the PWM components in the flux density are attenuated. As a consequence, the $\partial \mathbf{B} / \partial t$ decreases and so do the losses related to it. From Fig. 9, it can also be determined that the classic eddy-current iron losses change almost linearly with the fundamental frequency. Take as an example the set of results for a rotor frequency of $2 \mathrm{~Hz}$. The ratios of loss for 25,10 , and $5 \mathrm{~Hz}$ to the $50-\mathrm{Hz}$ case are $16 / 32=0.5,6 / 32 \approx 0.19$, and $3 / 32 \approx 0.093$, respectively, which corresponds approximately to the ratio between fundamental frequencies.

The same characteristics described above for the classic eddycurrent loss are also found for the anomalous losses, depicted in Fig. 10: 1) an almost linear behavior against the fundamental frequency and 2) an increase in magnitude as the load decreases. These characteristics are common to both types of losses as they share the same $\partial \mathbf{B} / \partial t$ term. Note that anomalous losses are one order of magnitude smaller than the classic eddy-current loss.

A frequency analysis of the magnetic flux density in some locations of the rotor was carried to explain the physical distribution of losses and their constitution in the frequency domain. The rotor FEs under study are shown in Fig. 1 (elements 6-12) and the frequency bands A-D are defined in the same way as for the stator. Fig. 11 shows the power loss density in the chosen elements at full-load condition.

For the sinusoidal case in any element, the power density in band B is not null as it contains important slotting harmonics. The location of these harmonics is given by (7) [18]

$$
\left[1-\nu(1-s)+n \frac{N_{s}}{P}(1-s)\right] f
$$

where

$s \quad$ slip;

$n$ air-gap permeance harmonic number;

$\nu \quad$ stator harmonic number;

$N_{s} \quad$ number of stator slots;

$P$ pole pairs;

$f \quad$ fundamental frequency $(\mathrm{Hz})$.

The most relevant slotting harmonics contained in this band are the combination of third, fourth, fifth, and sixth air-gap permeance harmonics $(n)$ with the fundamental component of the stator series $(\nu)$.

For frequency band A, all the elements in Fig. 11 show that the loss density scales in an approximately quadratic way with respect to the stator fundamental frequency: the losses at $25 \mathrm{~Hz}$ are approximately $1 / 4$ of the losses at $50 \mathrm{~Hz}$. This was unexpected because the fundamental frequency for the rotor laminations is the same for all the cases shown in this figure $(2.5 \mathrm{~Hz}$ at full load) and, therefore, the power loss density in band A should be about the same for either 25 or $50 \mathrm{~Hz}$. However, the slotting harmonic frequencies are higher at a fundamental frequency of $50 \mathrm{~Hz}$ than at $25 \mathrm{~Hz}$ and account for the increase in losses.

For the 25-Hz PWM case, the losses in band $\mathrm{C}$ are higher than those in band B for elements 6,8 , and 9. This follows the same principle explained earlier for the stator classic eddy-current losses: at low modulation indexes, the sidebands around the second multiple of the switching frequency are larger than those around the first multiple of the switching frequency. This plays a crucial role in the power distribution along the frequency spectrum.

The slot bridge (element 6) has the highest losses of the set of elements chosen in the rotor. The tip of the rotor tooth (element 7) exhibits losses of the same order as the bridge; however, the power loss in the upper region of the spectrum is not as significant.

It is important to note that the magnitude of the power loss density decreases rapidly when moving from the rotor surface: from order $10^{5} \mathrm{~W} / \mathrm{m}^{2}$ in elements 6 and 7 , it decays by one order of magnitude in element 8 , which is located in the iron at the head of the bar. By the middle section of the tooth the power loss density is less than $200 \mathrm{~W} / \mathrm{m}^{2}$ and in the inner section of the rotor (elements 11 and 12) the loss density is less than $25 \mathrm{~W} / \mathrm{m}^{2}$. 


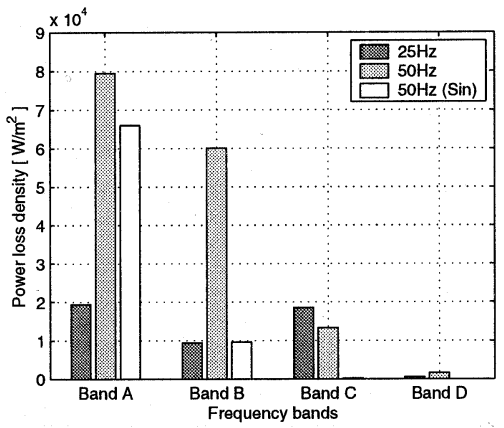

(a)

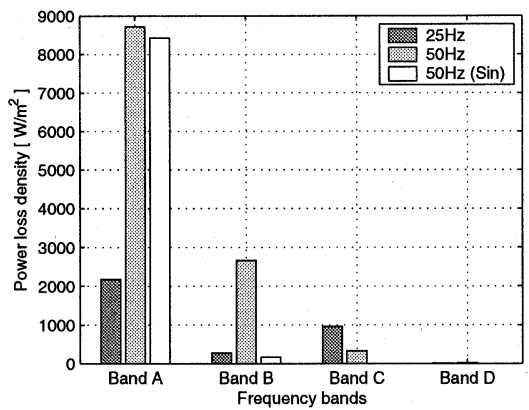

(c)

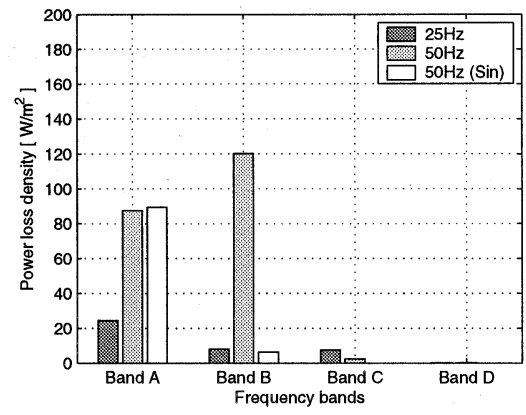

(e)

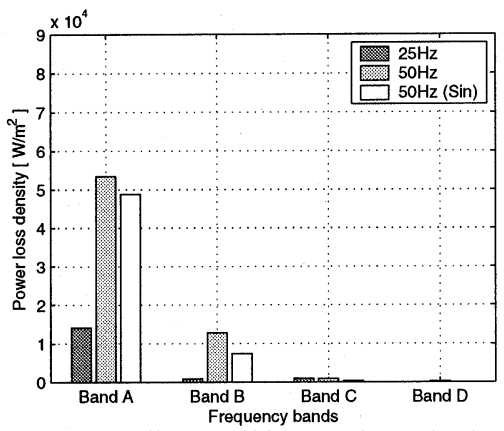

(b)

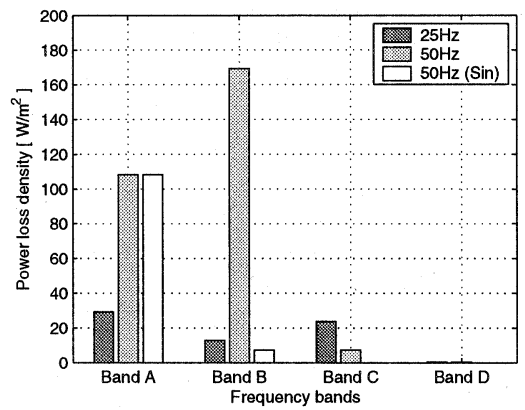

(d)

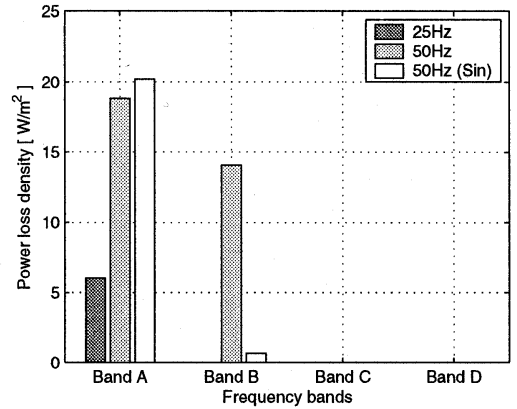

(f)

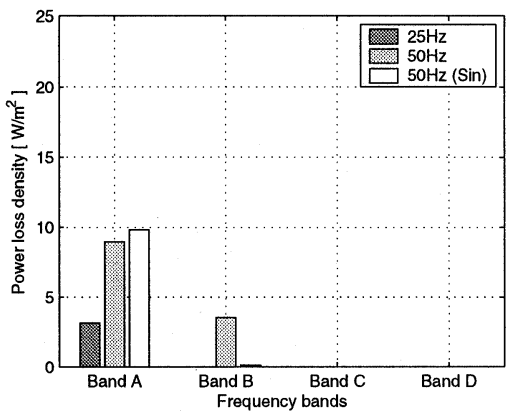

(g)

Fig. 11. Frequency partition of the power loss density in various elements of the rotor mesh. (a) Element 6: slot bridge. (b) Element 7: rotor tooth tip. (c) Element 8: bar head. (d) Element 9: Upper section of tooth. (e) Element 10: middle section of tooth. (f) Element 11: bottom of bar iron. (g) Element 12: inner core.

The power loss density in band $\mathrm{B}$ in elements 10 and 11 indicates that the PWM-related flux density is not restricted to the rotor surface as it is the case of some slotting harmonics [18] (those of high pole number). The losses due to PWM-related frequencies in the inner core of the rotor are comparable or larger than the losses in frequency band A.

As with the stator, band D was included in the analysis to show how little loss should be expected in the region of the third multiple of the switching frequency.

\section{Ohmic Power Loss}

Ohmic losses are relatively straightforward to calculate under the assumptions that the stator and rotor resistances are known and constant. The ohmic power losses calculated for the stator and rotor at full load and light load are presented in Table I.

As expected, when the motor is running at light load, the ohmic losses are low, especially for the rotor. From this table, it can also be noticed that the difference in loss between the sinusoidal and PWM excitation at $50 \mathrm{~Hz}$ is marginal. This is hardly 
TABLE I

OHMIC POWER LOSSES (WATTS)

\begin{tabular}{l|cccc}
\hline \multirow{2}{*}{$\begin{array}{l}\text { Fundamental } \\
\text { frequency }\end{array}$} & \multicolumn{2}{|c}{ Stator } & \multicolumn{2}{c}{ Rotor } \\
\hline $25 \mathrm{~Hz}(\mathrm{PWM})$ & 212.9 & 96.9 & 116.9 & 6.9 \\
$50 \mathrm{~Hz}(\mathrm{PWM})$ & 227.4 & 84.6 & 121.5 & 6.9 \\
$50 \mathrm{~Hz}(\mathrm{Sin})$ & 225.6 & 83.7 & 120.4 & 6.9 \\
\hline
\end{tabular}

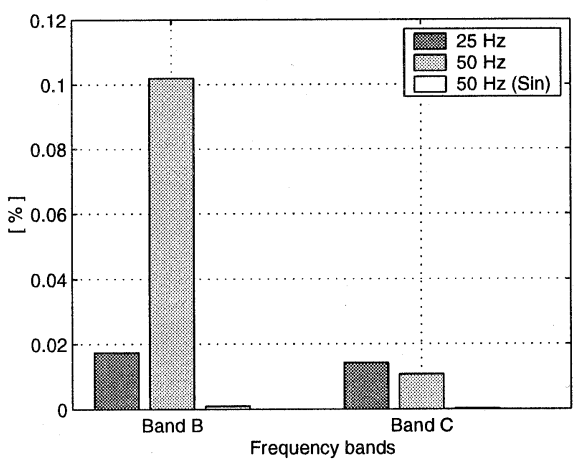

Fig. 12. Full-load stator ohmic loss.

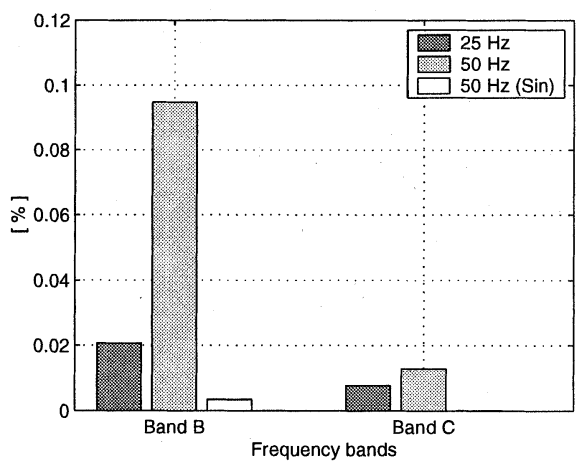

Fig. 13. Light-load stator ohmic loss.

a surprise as these two simulations were designed to have the same fundamental voltage and, therefore, about the same fundamental current.

The extra ohmic power loss introduced by the PWM frequency components is small, as the following analysis in the frequency domain shows. The frequency spectrum was divided in the same bands A-D as for the iron loss discussion and the power loss was calculated for each band.

For the stator, the findings are that above $99.86 \%$ of the losses take place in frequency band A for any of the fundamental frequencies presented in Table I, for both full- and light-load conditions. This indicates how little the bands associated to PWM action contribute to the total ohmic loss. The percentage of the loss dissipation for bands B and C are shown in Figs. 12 and 13, for the full-load and light-load conditions, respectively. It must be said that these two plots are very similar to each other, suggesting that the percentage distribution of losses in the stator is very much independent of the machine loading. Although the

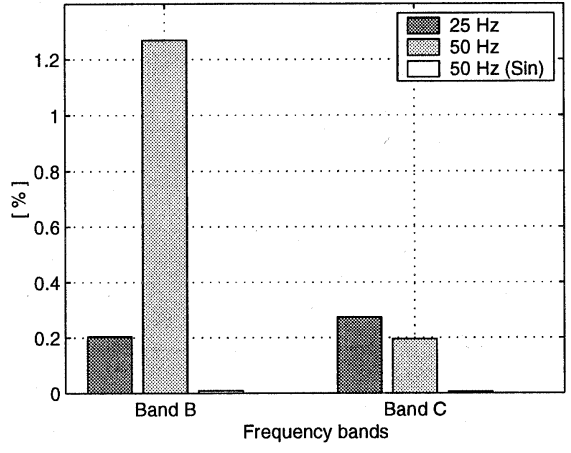

Fig. 14. Full-load rotor ohmic loss

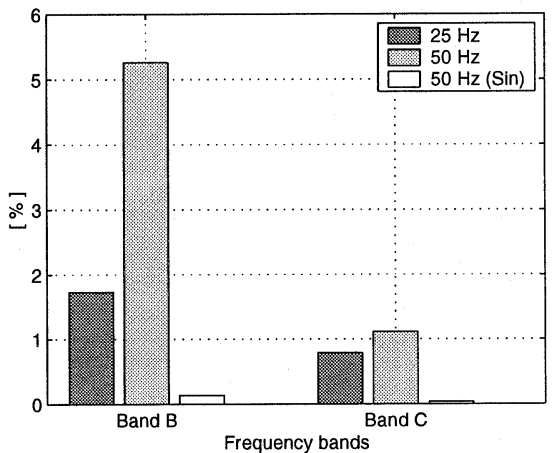

Fig. 15. Light-load rotor ohmic loss.

effect of the PWM-related frequencies can be noticed in these figures, the power loss contained in bands B, C, and D is quantitatively unimportant as they account for less than $0.16 \%$ of the total stator loss (losses in band D were found too small to be shown in Figs. 12 and 13).

For the rotor, above $94.72 \%$ of the ohmic losses are found in band A. Although this amount is slightly lower than for the stator, it is still large enough to say that very little loss occurs outside this band. Figs. 14 and 15 depict the contribution of bands $\mathrm{B}$ and $\mathrm{C}$ to the total ohmic loss in the rotor, at full and light load, respectively. By comparing the scale on the axes of these figures with the ones corresponding to the stator it is noted that the contribution of bands $\mathrm{B}$ and $\mathrm{C}$ is ten times larger for the rotor than for the stator.

It should be noted from the plots in Figs. 14 and 15 that the percentage of loss contained in bands $\mathrm{B}$ to $\mathrm{C}$ at light load increases almost by a factor of five with respect to the full-load case. This phenomenon is easily explained by considering that the fundamental rotor current decreases quickly when the motor load decreases, therefore, the harmonic content becomes relatively more important. This indicates that, however small, the PWM-related losses are more significant at light loads.

The most important remark to be obtained from the previous analysis is that the extra loss introduced by the PWM-related frequency components has a much less severe impact on ohmic losses than on iron losses.

\section{EXPERIMENTAL VALIDATION}

The experimental machine was a specially built cage induction motor with the following characteristics: three phases, four 


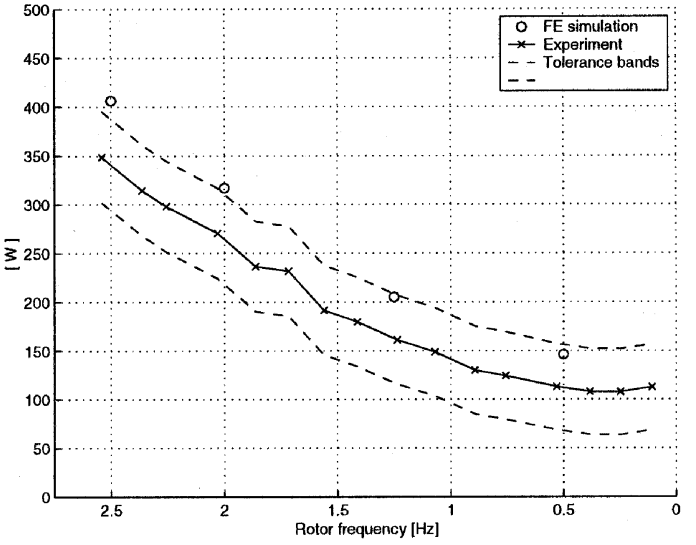

Fig. 16. Results at 50-Hz sinusoidal excitation.

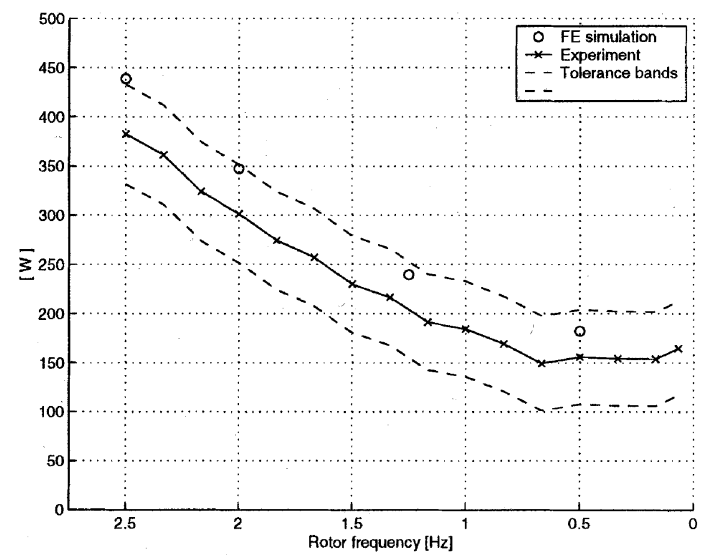

Fig. 17. Results at 50-Hz PWM excitation.

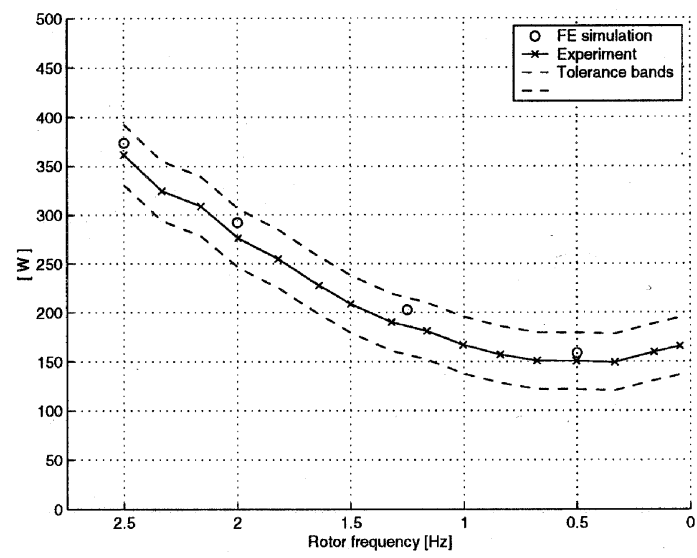

Fig. 18. Results at 25-Hz PWM excitation.

poles, $240 \mathrm{~V}$, delta connected, $2.1 \mathrm{~kW}, 1425 \mathrm{r} / \mathrm{min}, 0.8$ power factor, and rotor unskewed.

The experimental work was based on an input-output power measurement method. The induction motor is mechanically coupled to an independently excited dc machine which provides a load torque. The torque was manipulated through the field voltage applied to the dc machine and some external rheostats. The input power into the induction machine was measured by a PM3000A power analyzer connected between the power supply and the motor. The output power was calculated by measuring

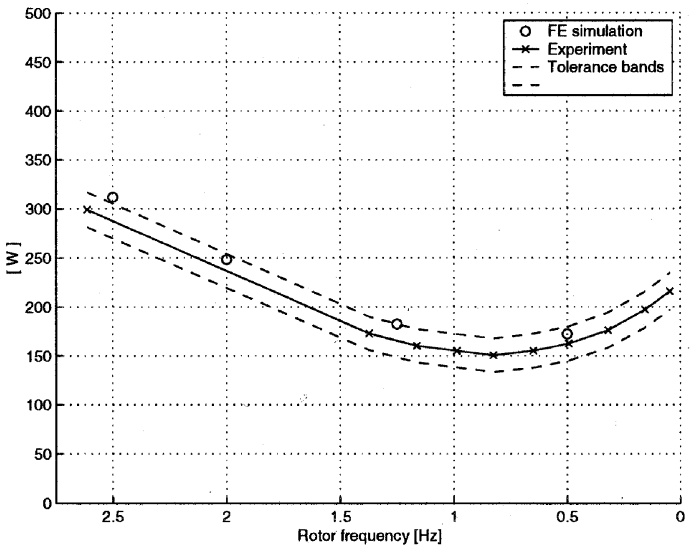

Fig. 19. Results at 10-Hz PWM excitation.

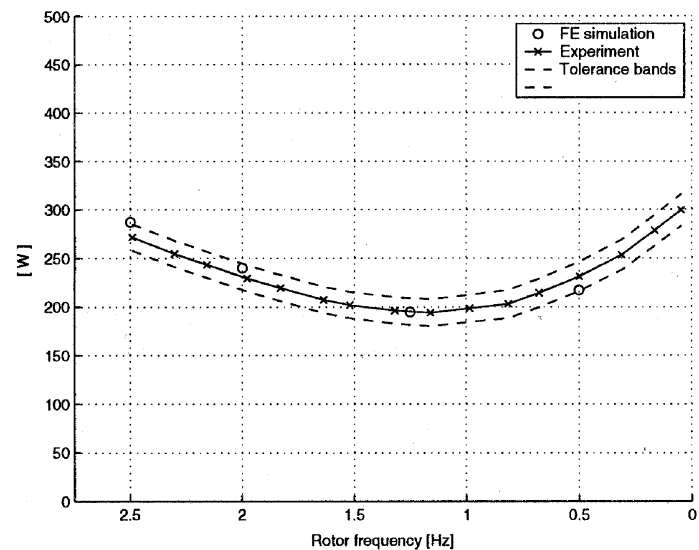

Fig. 20. Results at 5-Hz PWM excitation.

the torque between the two machines and the speed of the shaft. The torque was measured by means of a contactless sensor. Additional instrumentation in the test rig included a thermocouple buried in the stator winding which was used to correct the resistance values. The topology of the power converter used in this project was the standard voltage-source inverter (based on a 15-A 1200-V insulated gate bipolar transistor (IGBT) module, model BSM15GD120D2 by Siemens) without the front-end full-wave rectifier. Instead, the power for the dc link is provided by a variable-voltage dc power supply. The triggering signals for the power converter module are generated by a digital signal processor (DSP), which follows a sinusoidal regular-sampled PWM strategy.

The losses by windage and friction are subtracted from the measured power loss $\left(P_{\text {in }}-P_{\text {out }}\right)$ leaving as a result the combination of ohmic and iron losses. This combined power loss is compared against the simulation results presented in the previous section. Figs. 16-20 show this comparison for fundamental frequencies of 50, 25, 10, and $5 \mathrm{~Hz}$. Fig. 16 shows the case of a $50-\mathrm{Hz}$ sinusoidal power supply and the rest correspond to PWM excitation.

The dashed lines in all these figures are the measurement tolerance errors, which take into consideration the limitations of the power analyzer and the torque sensor. In the worst case scenario, the tolerance bands represent an accuracy of $2.16 \%$ of the rated input power. By using a calorimetric test rig this figure 
may be improved to the order of $0.14 \%$ [19], at the expense of a longer development time and a higher installation cost.

The torque sensor accuracy $( \pm 0.25 \mathrm{~N} \cdot \mathrm{m})$ accounts for more than $80 \%$ of the tolerance bands at full load and almost $90 \%$ at light load. This strong dependency of the tolerance bands on the torque measurement makes the width of the bands speed dependent.

In all cases presented in this section, from 50 to $5 \mathrm{~Hz}$ and from no load to light load, the difference between the measured and simulated values is within $2.5 \%$ of the rated input power. The fact that this difference scales at the same pace as the width of the tolerance bands suggests that the error might be in the calibration of the torque-sensing unit.

An interesting comparison arises from Figs. 16 and $17(50-\mathrm{Hz}$ sinusoidal excitation and 50-Hz PWM excitation cases, respectively): at full load, the experimental results are about $341 \mathrm{~W}$ for the first case and $383 \mathrm{~W}$ for the latter. This represents an increment in loss dissipation of $12 \%$. Given that the ohmic loss analysis has revealed that the PWM-related frequencies add very little loss, this $12 \%$ is mainly attributed to iron losses. At a light-load condition the power loss increment is larger at $40 \%$.

An important feature in every plot is that the difference between the simulated values and the experimental results is slightly larger at full load, compared with a light-load condition. This is attributed to a mismatch between the FE program input data and the real machine characteristics in some aspects, that could include: the laminations reluctivity, the operating rotor temperature, and the conductivity of the rotor bars.

At a fundamental frequency of $10 \mathrm{~Hz}$, no torque reading was taken between a slip 2.5 and $1.3 \mathrm{~Hz}$ due to a mechanical resonance found in the test rig.

At low frequencies it is noticeable that the power losses decrease from full load up to a certain slip ( 0.8 for the $10-\mathrm{Hz}$ case in Fig. 19 and 1.2 for the 5-Hz case in Fig. 20) and then they increase again toward the light-load region. This behavior is due to ohmic losses. While the minimum rotor current always takes place at the synchronous speed, the minimum stator current occurs always at a subsynchronous speed. The exact point of this minimum depends on the value for the different reactances and resistances of the motor. At rated frequency this minimum is usually located very close to the synchronous speed. When the fundamental frequency decreases, the reactances scale linearly with it but not the stator resistance, changing the relative magnitude between the resistive and reactive parts of the input impedance. The final outcome is that the point where the minimum stator current occurs moves away from the synchronous speed. This phenomenon can be observed even in linear motor models, where no iron saturation is considered. When saturation is put in place, the minimum point occurs even further away from the synchronous speed. In terms of power loss, which are predominantly ohmic at low frequencies, the minimum loss point will occur at approximately the same operating point as the minimum stator current. It must be observed that this phenomenon may be attenuated with closed-loop controllers (such as vector control or direct torque control) which regulate the magnitude of the magnetizing current independently of the load of the machine; however, the open-loop V/F control strategy implemented in this experiment compensates for the voltage drop across the resistance against the fundamental frequency but it does not provide any compensation against the load of the machine.

The FE simulations are in good agreement with the experimental results. This is attributed to the assurance of a firm numerical convergence in every time step and the cross check of the results coherence in the time and frequency domains.

\section{CONClusions}

FE analysis coupled to a circuit model of an inverter has been used to study the power loss distribution in an induction machine under regular-sampled PWM excitation for various operating conditions. Emphasis was put on iron losses as they are still a challenge in machine design and the impact of PWM-related frequency components is more severe on them than on ohmic losses. The findings are as follows.

When a fundamental frequency lower than the base frequency is applied, the modulation index is reduced to maintain the mutual flux constant. A low modulation index increases the frequency components around the second multiple of the switching frequency. These have an important impact on iron losses.

The behavior of classic eddy-current and anomalous losses as functions of the fundamental frequency is as expected but the contribution of the high-order PWM-related harmonics is dominant.

The PWM-related frequency components of the flux density are distributed across the motor laminations in a different pattern to those at fundamental frequency.

Both the presence of high-frequency fields and their nonuniform special distribution, mean that modeling iron losses in some applications as a three-term polynomial of the fundamental frequency $\left(P_{\text {loss }}=a F_{\text {fund }}+b F_{\text {fund }}^{2}+c F_{\text {fund }}^{3 / 2}\right.$, where $a, b$ and $c$ are constants) may be right for the wrong reasons. If $a, b$, and $c$ are properly chosen, this polynomial may model the iron losses through curve fitting. This might lead to the right numeric result but it may have no relation to the actual loss phenomena. The association of the linear term with the hysteresis loss, the quadratic term with the classic eddy-current loss, and the last term with the anomalous loss no longer holds true in these circumstances.

The almost-linear increase of the total iron losses against the fundamental frequency that has been found in this work is only a particular case and may not be valid as a general rule. Iron loss modeling in inverter-driven induction motors is a complex problem in which the fundamental frequency, the load of the machine, the modulation index and the PWM modulation strategy are all important factors shaping the iron loss behavior.

LCF is only an approximation to account for the minor loops in the hysteresis curve but it has been sufficient to indicate the significance of these minor loops in the iron loss calculation, particularly in the rotor. The values for the LCF obtained for the rotor in the current work are much greater than any value quoted in Lavers' paper. This indicates that the rotor contains flux waveforms with a much higher distortion than any of the cases presented in [13]. This underlines the importance of further research to improve the approximations for the minor loops contained in a BH-curve. 
The simulations have been validated by experimental data wherever possible, but it is not physically feasible to measure all the loss components separately. Only simulation can thoroughly segregate iron power loss into different types, different frequency bands, and different physical distribution.

\section{ACKNOWLEDGMENT}

The authors are thankful to Dr. C. McClay for her invaluable advice during this work.

\section{REFERENCES}

[1] P. Bertoldi, A. de Almeida, and F. E. Hugh, Energy Efficiency Improvements in Electric Motors and Drives, 1st ed. Berlin, Germany: Springer-Verlag, 2000

[2] K. Kytömäki, A. Arkkio, and A. Suontausta, "Validity of conventional and modern methods of no-load loss calculation in asynchronous machines-statistic approach," in Proc. IEE Int. Conf. Electrical Machines and Drives, Sept. 1997, vol. 1, pp. 185-189.

[3] B. A. Behrend, The Induction Motor, 2nd ed. New York: McGrawHill, 1921.

[4] P. L. Alger, Induction Machines-Their Behavior and Uses, 2nd ed. New York: Gordon and Breach, 1995

[5] R. C. Healey, S. Williamson, and A. Smith, "Improved cage rotor models for vector controlled induction motors," IEEE Trans. Ind. Applicat., vol. 31, pp. 812-822, July/Aug. 1995.

[6] G. C. D. Sousa, B. K. Bose, J. Cleland, R. J. Spiegel, and J. P. Chappell, "Loss modeling of converter induction machine system for variable-speed drive," in Proc. IEEE Conf. Industrial Electronics, Control, Instrumentation and Automation, 1992, vol. 1, pp. 114-120.

[7] N. E. Hildebrand and H. Roehrdanz, "Losses in three-phase induction machines fed by pwm converter," IEEE Trans. Energy Conversion, vol. 16, pp. 228-233, Sept. 2001.

[8] S. Williamson, L. H. Lim, and M. J. Robinson, "Finite element models for cage induction motor analysis," IEEE Trans. Ind. Applicat., vol. 26, pp. 1007-1017, Nov./Dec. 1990.

[9] C. I. McClay and S. Williamson, "Influence of rotor skew on cage motor losses," Proc. IEE-Elect. Power Applicat., vol. 145, pp. 414-422, Sept. 1998.

[10] C. A. Hernandez-Aramburo, T. C. Green, and C. I. McClay, "Integrated simulation of an inverter driven induction motor," in Proc. IEE Int. Conf. Power Electronics and Variable Speed Drives, 2000, pp. 560-565.

[11] P. Beckley, "Modern steels for transformers and machines," IEE Power Eng. J., vol. XIII, pp. 190-200, Aug. 1999.

[12] M. A. Mueller, S. Williamson, T. J. Flack, K. Atallah, B. Baholo, D. Howe, and P. H. Mellor, "Calculation of iron losses from time-stepped finite-element models of cage induction machines," in Proc. IEE Int. Conf. Electrical Machines and Drives, Sept. 1995, vol. 1, pp. 88-92.

[13] J. Lavers, P. Biringer, and H. Hollitscher, "A simple method of estimating the minor loop hysteresis loss in thin laminations," IEEE Trans. Magn., vol. MAG-14, pp. 386-388, Sept. 1978.

[14] R. Kaczmarek, M. Amar, and F. Protat, "Iron loss under pwm voltage supply on Epstein frame and induction motor core," IEEE Trans. Magn., vol. 32, pp. 189-194, Jan. 1996.

[15] L. R. Dupre, R. Van Keer, and J. A. A. Melkebeek, "An iron loss model for electrical machines using the Preisach theory," IEEE Trans. Magn., vol. 33, pp. 4158-4160, Sept. 1997.

[16] L. R. Dupre, J. J. Gyselinck, and J. A. A. Melkebeek, "Complementary finite element methods in 2D magnetics taking into account a vector Preisach model," IEEE Trans. Magn., vol. 34, pp. 3048-3051, Sept. 1998.
[17] C. I. McClay and G. T. Van der Thorn, "A comparison of time-stepped finite element techniques for the calculation of losses in cage induction motors," in Proc. IEE Int. Conf. Electrical Machines and Drives, vol. 1, Sept. 1999, pp. 35-39.

[18] C. I. McClay, "Efficiency improvement of cage induction motors," Ph.D. dissertation, St. John's College, Univ. Cambridge, Cambridge, U.K., Feb. 1996.

[19] K. J. Bradley, A. Ferrah, R. Magill, J. C. Clare, P. Wheeler, and P. Sewell, "Improvements to precision measurement of stray load loss by calorimeter," in Proc. IEE Int. Conf. Electrical Machines and Drives, Sept. 1999, pp. 189-193.

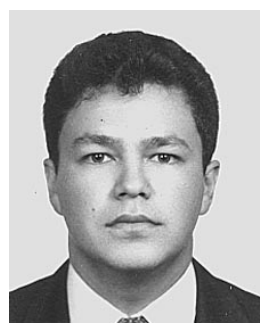

Carlos A. Hernandez-Aramburo (M'03) received the B.Sc. and M.Sc. degrees from the Universidad de las Americas, Puebla, Mexico, in 1994 and 1998, respectively, and the Ph.D. degree from Imperial College, London, U.K., in 2003.

$\mathrm{He}$ is currently a Research Associate in the Control and Power Research Group at Imperial College. His professional interests include electrical machines and drives, active power filters, and technology for distributed generation.

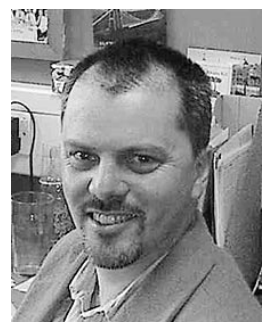

Tim C. Green (M'89-SM'02) received the B.Sc. (Eng.) degree with first class honors from Imperial College, London, U.K., in 1986, and the Ph.D. degree from Heriot-Watt University, Edinburgh, U.K., in 1990, both in electrical engineering.

He was at Heriot-Watt University until 1994. $\mathrm{He}$ is currently a Senior Lecturer and a member of the Control and Power Research Group at Imperial College. His research interests include power engineering, covering distributed generation, micro-grids, power quality, active power filters, FACTS technology, control of power systems using FACTS, devices, and machine drives.

Dr. Green is a Chartered Engineer in the U.K. and a Member of the Institution of Electrical Engineers, U.K

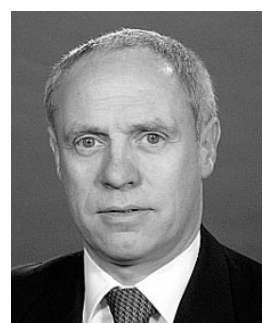

Sandy Smith (M'89-SM'02) received the B.Sc. (Eng.) and Ph.D. degrees from Aberdeen University, Aberdeen, U.K., in 1977 and 1980, respectively.

Academic appointments followed at Imperial College (1983-1990) and Cambridge University (1990-1997). In 1997, he joined Invensys Brook Crompton (formerly Brook Crompton) as Head of Research responsible for motor technology. In 2000, he was appointed Senior Lecturer at the University of Manchester Institute of Science and Technology, Manchester, U.K., where he was promoted to Reader in Electrical Machines in 2002. He became the Head of the Power Conversion Group in August 2002.

Dr. Smith is Executive Chairman of the Power Conversion and Applications Professional Network of the Institution of Electrical Engineers, U.K. 